\title{
Model Predictive Control of Stochastic Linear Systems with Probability Constraints
}

\author{
C.F. Caruntu, C.C. Velandia-Cardenas, X. Liu, A.N. Vargas
}

\section{Constantin F. Caruntu}

Department of Automatic Control and Applied Informatics,

Gheorghe Asachi Technical University of Iasi, Str. Prof. D. Mangeron no. 27, Iasi, Romania.

\section{Cristian C. Velandia-Cardenas}

Research Group MEM, Faculty of Electronic Engineering,

Universidad Santo Tomás, Cra. 9 no. 51-11, Bogotá, Colombia.

\section{Xinghua Liu}

Department of Electrical Engineering,

School of Automation and Information Engineering,

Xi'an University of Technology, Xian 710048, China.

\author{
Alessandro N. Vargas* \\ Universidade Tecnológica Federal do Paraná, UTFPR, \\ Av. Alberto Carazzai 1640, 86300-000 Cornelio Procópio-PR, Brazil. \\ *Corresponding author: avargas@utfpr.edu.br
}

\begin{abstract}
This paper presents a strategy for computing model predictive control of linear Gaussian noise systems with probability constraints. As usual, constraints are taken on the system state and control input. The novelty relies on setting bounds on the underlying cumulative probability distribution, and showing that the model predictive control can be computed in an efficient manner through these novel boundsan application confirms this assertion. Indeed real-time experiments were carried out to control a direct current (DC) motor. The corresponding data show the effectiveness and usefulness of the approach.
\end{abstract}

Keywords: probability constraints, stochastic systems, linear systems, control.

\section{Introduction}

Engineering applications show that constraints are not always met with probability one [4]. It is then reasonable to account constraints, together with performance indicators, under certain probability levels, e.g. [9] - this setup is referred to as model predictive control (MPC) with probability constraints, as detailed next.

Probability constraints on linear systems constitute a research topic that has attracted attention in recent years, in particular when it is combined with the MPC method, see for instance $[2,5,10,15,20,25]$, just to name a few. In this scenario, the model accounts additive disturbances $[5,15,20,25]$, multiplicative disturbances [2], and systems with mixed features; namely, systems with known:

1. probability distribution of the system output $[15,20]$;

2. Gaussian distribution and general probability distribution of the disturbance [5];

3. observable probability distribution of the disturbance at each time step [2];

4. covariance and mean of the inputs and outputs [10]. 
In particular, probabilistic constraints on inputs $[5,10,11]$, states $[2,5,10,11,25]$, and outputs $[15,20]$ were transformed into deterministic constraints on the process input.

To handle probability constraints is a task that the literature suggests the evaluation of element-wise probability constraints $[2,10,11,15,16,20,25]$; here, we follow the literature with a minor adaptation, that is, the element-wise evaluation yields nonlinear equations, difficult to compute.

To overcome this difficulty, we propose an upper bound for the underlying nonlinear equations. As a byproduct, we obtain linear matrix inequalities - computing linear matrix inequalities increases efficiency on solving optimization problems [4, Ch. 1].

The main contribution of this paper is to convert nonlinear equations, drawn from probability constraints, into linear matrix inequalities useful for real-time implementation. This assertion is illustrated in practice through the MPC applied in a direct current (DC) motor - experimental data are shown in Section 4. These findings illustrate the practical benefits of our approach.

The paper is organized as follows. Section 2 states the problem to be solved and the derived solution. Section 4 provides a performance evaluation of the proposed MPC with probability constraints based on the experimental results obtained on a direct current motor. Finally, concluding remarks are given in Section 5.

\section{Notation and basic definitions.}

$\mathbb{R}, \mathbb{R}_{+}, \mathbb{Z}$ and $\mathbb{Z}_{+}$are the real, non-negative real, integer and non-negative integer numbers. $\mathbb{Z}_{\geq c_{1}}$ and $\mathbb{Z}_{\left(c_{1}, c_{2}\right]}$ denote the sets $\left\{k \in \mathbb{Z}_{+} \mid k \geq c_{1}\right\}$ and $\left\{k \in \mathbb{Z}_{+} \mid c_{1}<k \leq c_{2}\right\}$, respectively, for some $c_{1}, c_{2} \in \mathbb{Z}_{+} \cdot\|\cdot\|_{\infty}$ denotes the infinity norm; given $x \in \mathbb{R}^{n}$ and $c \in \mathbb{R}$, by definition of the infinity norm, for $\|x\|_{\infty} \leq c$ to be satisfied, it is necessary and sufficient that $\pm[x]_{j} \leq c$ for all $j \in \mathbb{Z}_{[1, n]}$. $I_{n}$ is the identity matrix of order $n$ and $M^{\prime}$ denotes the transpose of $M$. $P[A]$ denotes the probability of event $A$ and $p(x)$ denotes the probability distribution function of random variable $x . \bar{x}$ denotes the mean of the random variable $x$ and $S_{x}$ denotes its covariance.

\section{Problem statement}

For some fixed and filtered probability space $(\Omega, \mathcal{F}, P)$, consider the following discrete-time linear time invariant system

$$
x_{k+1}=A x_{k}+B u_{k}+H w_{k}, \quad k \in \mathbb{Z}_{+},
$$

where $x_{k}$ denotes an $n$-dimensional system state, $u_{k}$ represents an $m$-dimensional control input, and $w_{k}$ represents a $q$-dimensional noise or disturbance driven by an independent and identically distributed (i.i.d.) Gaussian process. It is assumed that the initial state $x_{0}$ is a Gaussian random variable uncorrelated with $w_{k}$.

Although it has been intensively studied over the last decades, (1) presents so far some interesting challenges. For instance, controlling (1) under probabilistic constraints remains an open problem [9].

The main contribution of this paper lies in showing a condition for controlling the system (1) under probabilistic constraints. We revisit some results from the literature, and show how to convert them into linear matrix inequalities, a useful scheme for numerical evaluations.

To turn our contribution clear, we present some notation borrowed from [3]. Stacking the 
elements of (1) for $N$ steps in the form

$$
\mathbb{X}=\left[\begin{array}{c}
x_{1} \\
x_{2} \\
\vdots \\
x_{N}
\end{array}\right], \quad \mathbb{U}=\left[\begin{array}{c}
u_{0} \\
u_{1} \\
\vdots \\
u_{N-1}
\end{array}\right], \quad \mathbb{W}=\left[\begin{array}{c}
w_{1} \\
w_{2} \\
\vdots \\
w_{N}
\end{array}\right],
$$

we obtain the $N n$-dimensional vector representation

$$
\mathbb{X}=G_{x x} x_{0}+G_{x u} \mathbb{U}+G_{x w} \mathbb{W} .
$$

where the matrices $G_{x x}, G_{x u}$ and $G_{x w}$ are fixed, and their entries depend only on $A, B$, and $H$. Here, $N$ is called prediction horizon. Note that $\mathbb{X}$ in (3) is a multivariate normal random vector, a direct consequence of the i.i.d. Gaussian assumption on $\mathbb{W}$.

Set $\overline{\mathbb{X}}$ as the mean value of $\mathbb{X}$ and let $S_{\mathbb{X}}$ be its covariance, both given by [3]

$$
\begin{aligned}
\overline{\mathbb{X}} & =G_{x x} \bar{x}_{0}+G_{x u} \mathbb{U}+G_{x w} \overline{\mathbb{W}} \\
S_{\mathbb{X}} & =G_{x x} S_{x_{0}} G_{x x}^{\prime}+G_{x w} S_{\mathbb{W}} G_{x w}^{\prime} .
\end{aligned}
$$

The probabilistic constraints to be imposed on (1) are defined as follows. Let $F_{X}$ be the set made up by the intersection of $M$ linear inequality constraints, i.e.,

$$
F_{X}:=\bigcap_{i=1}^{M}\left\{\mathbb{X}: a_{i}^{\prime} \mathbb{X} \leq b_{i}\right\},
$$

where $a_{i}$ and $b_{i}, i=1, \ldots, M$, represent vectors on $\mathbb{R}^{n N}$ and $\mathbb{R}$, respectively. Moreover, consider $F_{U}$ as a set defined in similar fashion.

Assume for the moment that a given functional $J: F_{X} \times F_{U} \mapsto \mathbb{R}_{+}$, convex with respect to its arguments, is associated with (1). Hereafter, this functional is referred to as cost function.

Problem 1. Given some $\delta \in \mathbb{R}_{(0,1)}$, find a solution for the next optimization problem.

$$
\begin{array}{rl}
\min _{\mathbb{U} \in F_{U}} & J(\overline{\mathbb{X}}, \mathbb{U}) \\
\text { s.t. } & \operatorname{Pr}\left[\mathbb{X} \notin F_{X}\right] \leq \delta, \\
\mathbb{X}= & G_{x x} x_{0}+G_{x u} \mathbb{U}+G_{x w} \mathbb{W}
\end{array}
$$

As a matter of fact, Problem 1 means that the control input minimizes the cost function while ensuring that the system state may leave the feasible region with a probability less or equal than $\delta$.

To the best of the authors' knowledge, Problem 1 remains unresolved. The difficulty to solve such a problem stems from the fact that the probability in (6) represents a value taken over by multivariate integrals, requiring their evaluation, a difficult task to accomplish $[12,17]$.

To avoid the evaluation of multivariate integrals, the authors of [3] suggest the element-wise evaluation of the set $F_{X}$ in probability; namely, find constants $\varepsilon_{i}$ 's such that $\sum_{i=1}^{M} \varepsilon_{i} \leq \delta$, and

$$
\operatorname{Pr}\left[a_{i}^{\prime} \mathbb{X}>b_{i}\right] \leq \varepsilon_{i}, \quad \forall i \in \mathbb{Z}_{[1, M]} .
$$

Note that $\varepsilon_{i}$ 's are slack variables, now in association with a univariate probabilistic inequality, which can be computed via the standard cumulative distribution function. Even so, despite the progress researchers have made so far, the problem still remains difficult to solve because checking (7) may be cumbersome from the numerical point of view, as detailed next. 


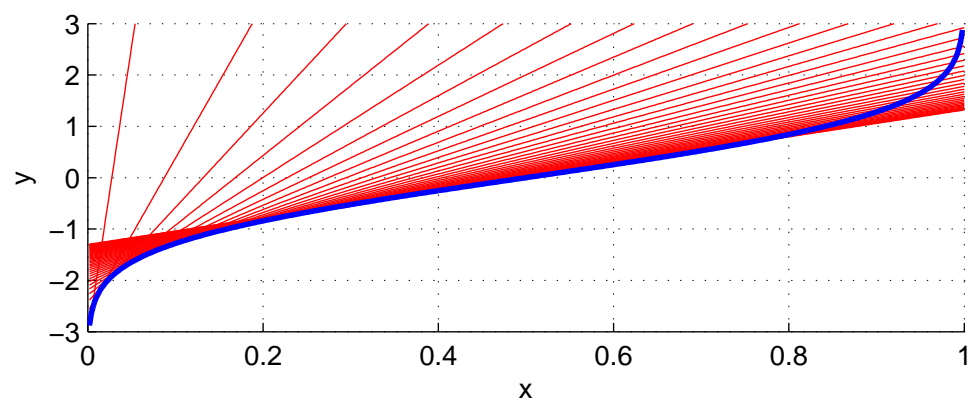

Figure 1: The inverse cumulative distribution function (thick line) and upper linear approximations (straight lines tangent to the icdf function).

Let $\operatorname{cdf}(\cdot)$ be the standard Gaussian cumulative distribution function

$$
\operatorname{cdf}(\gamma)=\frac{1}{\sqrt{2 \pi}} \int_{-\infty}^{\gamma} e^{-\frac{z^{2}}{2}} d z
$$

and let $\operatorname{icdf}(\cdot)$ be the corresponding inverse.

One can show that (7) holds if and only if both inequalities $\sum_{i=1}^{M} \varepsilon_{i} \leq \delta$ and

$$
\frac{b_{i}-a_{i}^{\prime} \overline{\mathbb{X}}}{s_{i}} \geq \operatorname{icdf}\left(1-\varepsilon_{i}\right), \quad \forall i \in \mathbb{Z}_{[1, M]},
$$

hold true, where $s_{1}, \ldots, s_{M}$, are fixed, positive constants.

Even though the inequalities in (9) are convex with respect to $\varepsilon_{i}$ 's, as shown in [3], finding a solution for (9) may be cumbersome - there is no closed, algebraic expression for computing a feasible solution for (9). Consequently, to evaluate (9), it is necessary to use iterative algorithms, usually slow for computing the point of convergence [1, Ch. 7]. But slow algorithms cannot be applied in some online control schemes, such as model predictive control $[6,7,14,19,24,26]$.

In order to speed up the computation of a feasible solution for (9), we convert (9) into an affine function so as to obtain linear matrix inequalities (LMI) - it is known that LMI solvers outperform classical convex optimization algorithms [4, Ch. 1]. The idea behind such conversion is now detailed.

As described in Fig. 1, the icdf function can be bounded from above by an affine function on some interval. This fact allows us to define an affine function $h(\cdot)$ such that

$$
h\left(1-\varepsilon_{i}\right) \geq \operatorname{icdf}\left(1-\varepsilon_{i}\right), \quad \forall i \in \mathbb{Z}_{[1, M]} .
$$

The novel condition hinges on checking whether the inequalities

$$
\frac{b_{i}-a_{i}^{\prime} \overline{\mathbb{X}}}{s_{i}} \geq h\left(1-\varepsilon_{i}\right), \quad \forall i \in \mathbb{Z}_{[1, M]},
$$

hold true. In the positive case (9) is assured; as a byproduct, we obtain $\mathbb{U}$ that suffices to (6) in Problem 1, the main condition pursued in this paper.

The main contribution of this paper is capable of showing (11). Yet derived from a simple idea of imposing upper bounds on the icdf function, the condition in (11) represents linear matrix inequalities, useful for real-time experiments - the paper shows a real-time application for a direct current motor (see Section 4). This application illustrates the practical benefits of our approach. 


\section{Model predictive control with probability constraints}

The problem we solve in this paper is presented in what follows. Define $y_{i}=a_{i}^{\prime} x$, and note that $y_{i}$ is a univariate Gaussian random variable with mean and variance given by

$$
\begin{aligned}
\bar{y}_{i} & =a_{i}^{\prime} G_{x x} \bar{x}_{0}+a_{i}^{\prime} G_{x u} \mathbb{U}+a_{i}^{\prime} G_{x w} \overline{\mathbb{W}} \\
S_{y_{i}} & =a_{i}^{\prime} G_{x x} S_{x_{0}} G_{x x}^{\prime} a_{i}+a_{i}^{\prime} G_{x w} S_{\mathbb{W}} G_{x w}^{\prime} a_{i} .
\end{aligned}
$$

Using the novel condition in (11), we recast the Problem 1 as the next problem.

Problem 2. Given some $\delta \in \mathbb{R}_{(0,1)}$, find the solution to the optimization problem

$$
\begin{aligned}
& \min _{\mathbb{U} \in F_{U}} J(\overline{\mathbb{X}}, \mathbb{U}) \\
& \text { s.t. } \quad \sum_{i=1}^{M} \varepsilon_{i} \leq \delta, \quad \frac{b_{i}-\bar{y}_{i}}{\sqrt{S_{y_{i}}}} \geq h\left(1-\varepsilon_{i}\right), \\
& \bar{y}_{i}=a_{i}^{\prime} G_{x x} \bar{x}_{0}+a_{i}^{\prime} G_{x u} \mathbb{U}+a_{i}^{\prime} G_{x w} \overline{\mathbb{W}}, \\
& S_{y_{i}}=a_{i}^{\prime} G_{x x} S_{x_{0}} G_{x x}^{\prime} a_{i}+a_{i}^{\prime} G_{x w} S_{\mathbb{W}} G_{x w}^{\prime} a_{i}, \forall i \in \mathbb{Z}_{[1, M]} .
\end{aligned}
$$

The next result is a direct consequence of the aforementioned facts.

Theorem 3. A feasible solution to Problem 2 is a feasible solution to Problem 1.

Proof: Recall that

$$
\begin{aligned}
\operatorname{Pr}\left[\mathbb{X} \notin F_{X}\right] & =\operatorname{Pr}\left[\mathbb{X} \notin \bigcap_{i=1}^{M}\left\{\mathbb{X}: a_{i}^{\prime} \mathbb{X} \leq b_{i}\right\}\right] \\
& =\operatorname{Pr}\left[\mathbb{X} \in \bigcup_{i=1}^{M}\left\{\mathbb{X}: a_{i}^{\prime} \mathbb{X}>b_{i}\right\}\right] .
\end{aligned}
$$

The rightmost term in (14) is bounded from above by $\sum_{i=1}^{M} \operatorname{Pr}\left[a_{i}^{\prime} \mathbb{X}>b_{i}\right]$ due to the Boole's inequality. When Problem 2 has a solution, we have from (10) and (13) that

$$
\frac{b_{i}-\bar{y}_{i}}{\sqrt{S_{y_{i}}}} \geq h\left(1-\varepsilon_{i}\right) \geq \operatorname{icdf}\left(1-\varepsilon_{i}\right), \quad \forall i \in \mathbb{Z}_{[1, M]},
$$

which shows (9). Both (9) and $\sum_{i=1}^{M} \varepsilon_{i} \leq \delta$ assure $\operatorname{Pr}\left[a_{i}^{\prime} \mathbb{X}>b_{i}\right] \leq \varepsilon_{i}$ for each $i=1, \ldots, M$ from (7), which in turn assures that $\operatorname{Pr}\left[\mathbb{X} \notin F_{X}\right] \leq \delta$. This argument completes the proof.

Remark 4. Theorem 3 assures that any optimal solution to Problem 2 is a sub-optimal solution to Problem 1-it means that the optimality of Problem 1 is lost; however, the sub-optimal strategy turns the problem tractable from the numerical viewpoint. Theorem 3 then contributes towards finding a solution to the so-far unsolved Problem 1.

Recall that the model predictive control (MPC) works under the receding horizon control principle [14, Ch. 1]; namely, the cost function $J(\cdot)$ in Problem 2 should be minimized at each time instant $k=k_{0}, \ldots, k_{1}$. The current input $u_{k}$ in (1) is obtained by determining the input sequence $\left\{\hat{u}_{k}, \ldots, \hat{u}_{k+N-1}\right\}$ that solves Problem 2, and next by setting $u_{k}=\hat{u}_{k}$. The remaining elements of the sequence are discarded, and this iterative procedure is repeated [14, Ch. 1].

The aforementioned iterative procedure, key for the MPC scheme, was implemented in a laboratory testbed as detailed in the next section. 


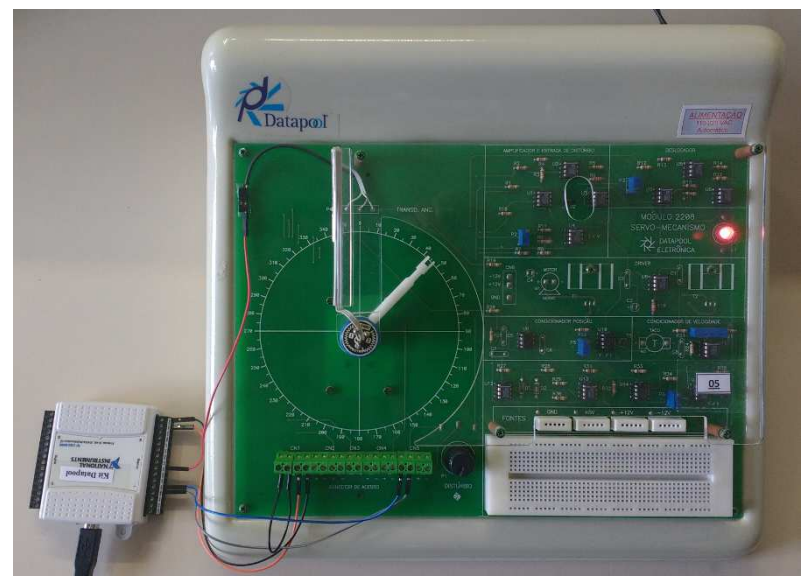

Figure 2: Equipment overview.

\section{Application of model predictive control to a direct current mo- tor subject to probability constraints}

The goal of this section is to illustrate the proposed model predictive control scheme with probability constraints. To do so, we present data collected from real-time experiments - the experiments were carried out in a laboratory tested designed to control a direct current (DC) motor.

\subsection{Identification}

The laboratory testbed was assembled with "Modulo 2208 servo-mecanismo", a DC motor device assembled by Datapool-Brazil $[18,21]$, which worked in association with a data-acquisition board (model USB-6008 DAQ) from National Instruments (see Fig. 2). The board was configured to work with fixed sampling time of $20 \mathrm{~ms}$.

The DC motor was equipped with a tachometer - it generated a voltage in the range $[0,5]$ $\mathrm{V}$ for an angular velocity in the range of $[0,26.17] \mathrm{rad} / \mathrm{s}$, in a linear relationship. Likewise, a current sensor generated the current consumed by the motor in the range $[0,5] \mathrm{A}$.

Pseudo random binary signals (PRBS) with amplitudes between 0 and $5 \mathrm{~V}$ were generated and applied in a sixth-order Butterworth low-pass filter with a cutoff frequency randomly chosen between $0.01 \mathrm{~Hz}$ and $70 \mathrm{~Hz}$. The resulting signal was then applied in the input of the DC motor, and the corresponding data was collected and stored.

Each experiment for sake of identification was performed in chunks of 60s, that is, each chunk contained three thousand points. The data were then split into smaller chunks, each with 1300 points. These chunks were used to obtain a model in the observability canonical form through the subspace identification method $[8,13]$. The canonical model was then modified to cope with the prediction-error minimization algorithm. Finally, the obtained model was validated through unused, uncorrelated data (see Fig. 3 for illustration of a sample). This procedure produced the model $(\approx 85 \%$ fitting $)$ :

$$
\begin{aligned}
{\left[\begin{array}{c}
\omega_{k+1} \\
i_{k+1}
\end{array}\right]=} & {\left[\begin{array}{cc}
0.9814 & 0.4582 \\
-0.0087 & 0.7667
\end{array}\right]\left[\begin{array}{c}
\omega_{k} \\
i_{k}
\end{array}\right] } \\
& +\left[\begin{array}{l}
0.0064 \\
0.0207
\end{array}\right]\left(u_{k}+d_{k}\right),
\end{aligned}
$$




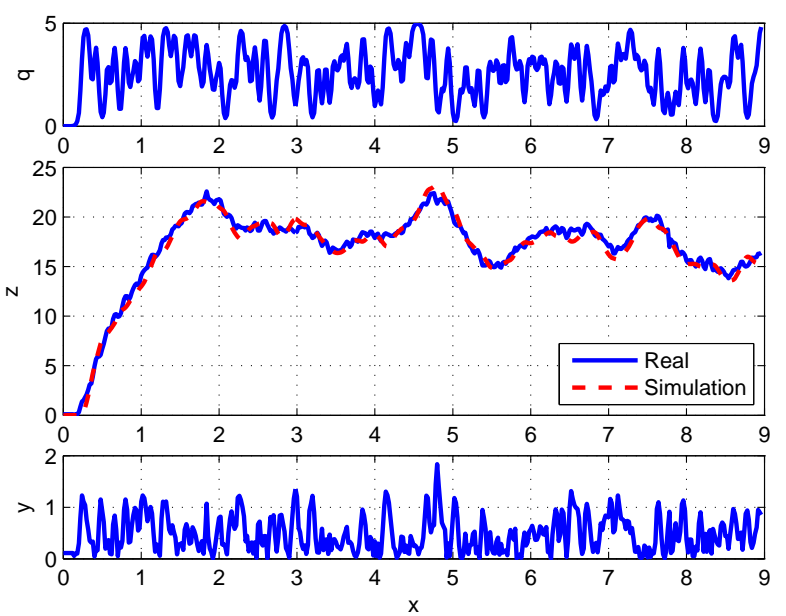

Figure 3: Real and simulated data: experiment suggests that the model attained for the DC motor seem appropriate for representing the real-time equipment.

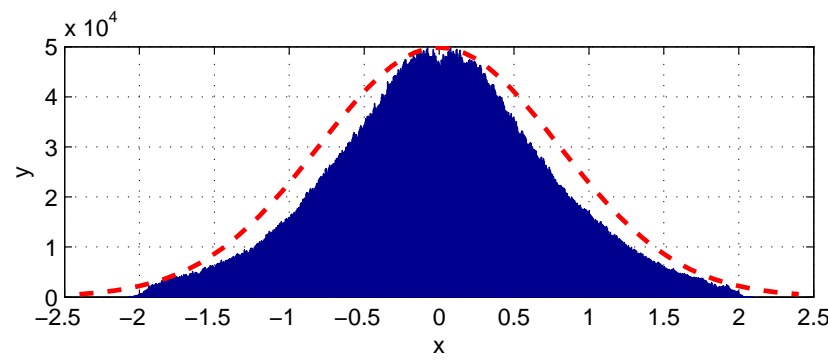

Figure 4: Histogram of disturbances. Dashed curve shows the Gaussian distribution.

where $\omega, i, u$, and $d$ represent the angular velocity of the shaft, the electrical current consumed by the motor, the armature voltage applied in the terminals of the DC motor, and disturbance, respectively.

The disturbance $d$ stands for an i.i.d. stochastic process under Gaussian probability distributionthe corresponding experimental data is summarized in Fig. 4.

\subsection{Probability constraints}

Armature voltage (control signal) that drove the motor was restricted by lower and upper bounds as

$$
u^{\min } \leq u_{k} \leq u^{\max },
$$

where $u^{\min }$ and $u^{\max }$ are the minimum and maximum voltages. The rotor angular velocity and the armature current were bounded as well, i.e.,

$$
\omega^{\min } \leq \omega_{k} \leq \omega^{\max }, \quad i^{\min } \leq i_{k}, \quad \text { and } \quad \operatorname{Pr}\left[i_{k} \geq i^{\max }\right] \leq \delta,
$$

where $\omega^{\min }, \omega^{\max }, i^{\min }$, and $i^{\max }$ denote the corresponding limits.

The control objective was to reach a desired value of the rotor angular velocity, say $\omega^{s s}$, while fulfilling the constraints given in (16) and (17). 


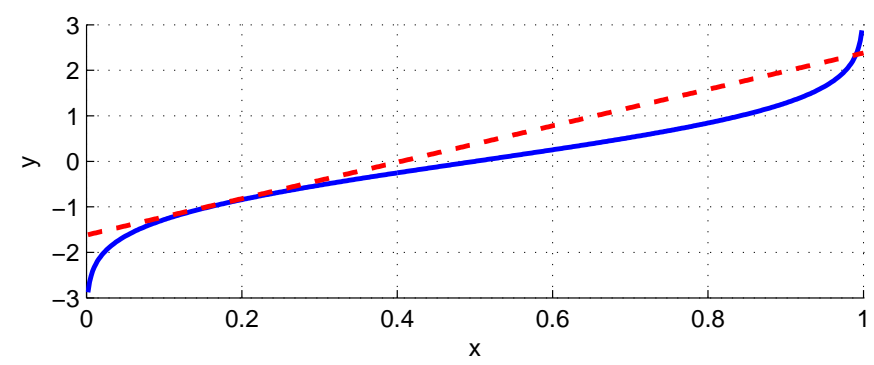

Figure 5: Inverse cumulative distribution function and its upper linear approximation (dashed line).

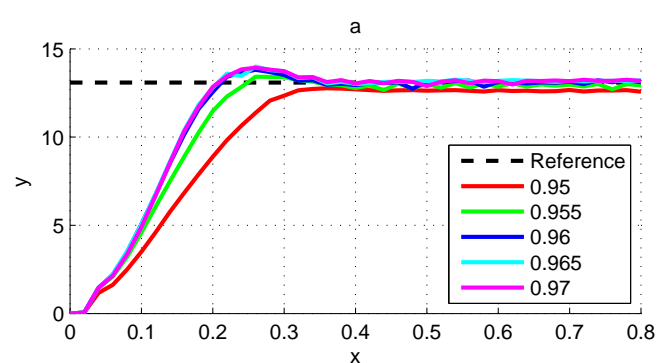

c

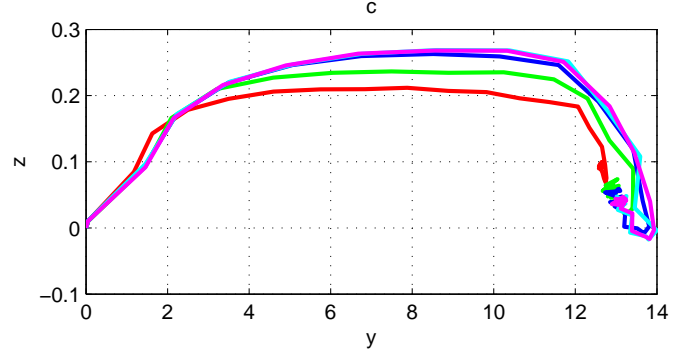

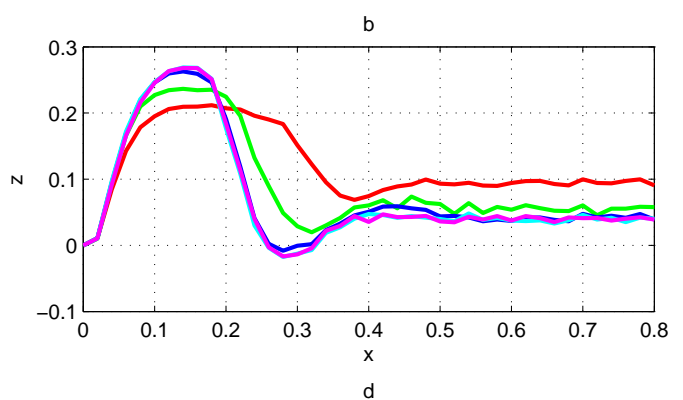

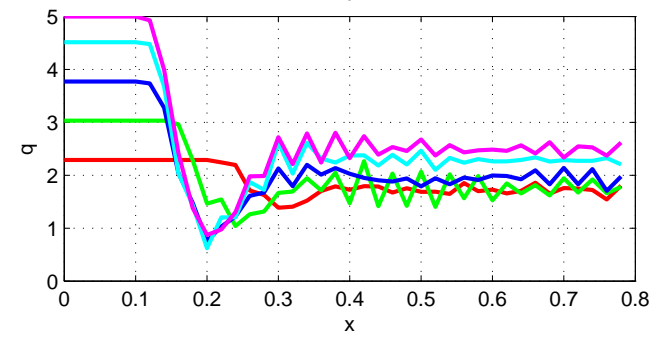

Figure 6: Data from real-time experiments. Problem 2 was evaluated for each $\delta=$ $0.95,0.955,0.96,0.965,0.97$, and every curve shows the mean of one-hundred sample paths. The curves show (a) angular velocity of the DC motor; (b) armature current of the DC motor; (c) phase-portrait of the system; and (d) armature voltage of the DC motor.

\subsection{Experimental results}

Recall that Problem 2 requires a linear approximation for the inverse cumulative distribution function. To fulfill this requirement, the linear approximation $h\left(1-\varepsilon_{i}\right)=2.38-4 \varepsilon_{i}$ was considered, valid for $\varepsilon_{i} \in[0.01,1)$ (see Fig. 5).

Associated with Problem 2, we consider the cost

$$
J(\overline{\mathbb{X}}, \mathbb{U})=1000\|\overline{\mathbb{X}}\|_{\infty}+2\|\mathbb{U}\|_{\infty} .
$$

Problem 2 was evaluated with the following additional parameters: $N=2, S_{x 0}=I_{2}$ and $S_{\mathbb{W}}=I_{2}$.

The limits for the constraints in (16) and (17) were set as $u^{\min }=0 \mathrm{~V}, u^{\max }=5 \mathrm{~V}, \omega^{\min }=0$ $\mathrm{rad} / \mathrm{s}, \omega^{\max }=26.17 \mathrm{rad} / \mathrm{s}, i^{\min }=0 \mathrm{~A}$ and $i^{\max }=0.38 \mathrm{~A}$. The reference for the angular velocity was fixed at $\omega^{s s}=13 \mathrm{rad} / \mathrm{s}$.

Problem 2 was evaluated with $\delta$ taking values $0.95,0.955,0.96,0.965$, and 0.97 , and every corresponding solution was checked in practice. Indeed, one-hundred experiments were conducted for each value of $\delta$ - the experimental outcome is subsumed under the curves shown Fig. 6. 
The curves in Fig. 6 show the statistical mean of the stored one-hundred samples. Fig. 6(a) shows the angular velocity of the DC motor; it can be observed that as $\delta$ decreases, settling time increases. Armature current is represented in Fig. 6(b), which shows that the upper bound on the current is satisfied and the maximum value of the current decreases when the value of $\delta$ decreases. Phase portrait of the process is illustrated in Fig. 6(c)-data suggest that the DC model be second moment stable [22]. The curves of the armature voltage (control input) in Fig. 6(d) suggest that as long as $\delta$ decreases, the upper bound on the armature voltage also decreases; as a result, $\delta$ successfully actuated as a constraint on the armature voltage.

In summary, the experimental data indicate that our approach seems to be useful to handle real-time applications subject to physical constraints.

\section{Conclusion}

The paper presents an easy-to-check method to handle linear stochastic systems subject to probability constraints. Here, constraints hinge upon Gaussian distributions. However, nonlinear equations arise naturally from that Gaussian-based evaluations, turning the problem difficult to solve. To overcome such a drawback, we have converted those nonlinear equations into simple-tocheck linear matrix inequalities (conversion guarantees only sub-optimal solutions, see Remark 4). The derived conversion shows appropriate for real-time applications - specially when fast algorithms are necessary — as illustrated in the paper.

\section{Acknowledgments}

This work was partially supported by the Thousand Talents Plan of Shaanxi Province for Young Professionals, and the Brazilian agencies FAPESP Grants 03/06736-7; CNPq Grant 304856/2007-0; and CAPES Grant Program PVE 88881.030423/2013-01.

\section{Bibliography}

[1] Bazaraa, M.S.; Sherali, H.D.; Shetty, C.M. (2006). Nonlinear programming: theory and algorithms, 3rd edn., Wiley-Interscience, New Jersey, 2006.

[2] Bernardini, D.; Bemporad, A. (2012). Stabilizing model predictive control of stochastic constrained linear systems, IEEE Trans. Autom. Control, 57, 1468-1480, 2012.

[3] Blackmore, L.; Ono, M. (2009). Convex chance constrained predictive control without sampling, In: AIAA Guidance, Navigation and Control Conference, Chicago, Illinois, USA, $1-17,2009$.

[4] Boyd, S.; El Ghaoui, L.; Feron, E.; Balakrishnan, V. (1994). Linear matrix inequalities in system and control theory, SIAM, Philadelphia, 1994.

[5] Cannon, M.; Kouvaritakis, B.; Rakovic, S.; Cheng Q. (2011). Stochastic tubes in model predictive control with probabilistic constraints, IEEE Trans. Autom. Control, 56, 194-200, 2011.

[6] Cao, G.; Lai, E.M.-K.; Alam, F. (2017). Gaussian process model predictive control of unknown non-linear systems, IET Control Theory Appl., 11, 703-713, 2017. 
[7] Caruntu, C.F.; Balau, A.E.; Lazar, M.; van den Bosch, P.P.J.; Di Cairano, S. (2016). Driveline oscillations damping: A tractable predictive control solution based on a piecewise affine model, Nonlinear Analysis: Hybrid Systems, 19, 168-185, 2016.

[8] Costa Junior, A.G.; Riul J.A.; Montenegro, P.H.M. (2016). Application of the subspace identification method using the N4SID technique for a robotic manipulator, IEEE Latin America Transactions, 14, 1588-1993, 2016.

[9] Farina, M.; Giulioni, L.; Scattolini, R. (2016). Stochastic linear model predictive control with chance constraints-A review, Journal of Process Control, 44, 53-67, 2016.

[10] Farina, M.; Giulioni, L.; Magni, L.; Scattolini, R. (2015). An approach to output-feedback MPC of stochastic linear discrete-time systems, Automatica, 55, 140-149, 2015.

[11] Farina, M.; Scattolini, R. (2016). Model predictive control of linear systems with multiplicative unbounded uncertainty and chance constraints, Automatica, 70, 258-265, 2016.

[12] Hashorva, E.; Hüsler, J. (2003). On multivariate Gaussian tails, Annals of the Institute of Statistical Mathematics, 55, 507-522, 2003.

[13] Katayama, T. (2005). Subspace methods for system identification, communications and control engineering, Springer-Verlag, London, 2005.

[14] Kwon, W.H., Han, S.H. (2005). Receding horizon control: model predictive control for state models, Springer-Verlag, New York, 2005.

[15] Li, P.; Wendt, M.; Wozny, G. (2002). A probabilistically constrained model predictive controller, Automatica, 38, 1171-1176, 2002.

[16] Li, J.W.; Li, D.W.; Xi, Y.G. (2017). $H_{\infty}$ predictive control with probability constraints for linear stochastic systems, IET Control Theory Appl., 11, 557-566, 2017.

[17] Lu, D.; Li, W.V. (2009). A note on multivariate Gaussian estimates, Journal of Mathematical Analysis and Applications,354, 704-707, 2009.

[18] Oliveira, R.C.L.; Vargas, A.N.; do Val, J.B.R.; Peres, P.L.D. (2014). Mode-independent $\mathrm{H}_{2}$-control of a DC motor modeled as a Markov jump linear system, IEEE Transactions on Control Systems Technology, 22, 1915-1919, 2014.

[19] Rubagotti, M.; Patrinos, P.; Guiggiani, A.; Bemporad, A. (2016). Real-time model predictive control based on dual gradient projection: theory and fixed-point FPGA implementation, Int. J. Robust Nonlinear Control, 26, 3292-3310, 2016.

[20] Schwarm, A.T.; Nikolaou, M. (1999). Chance-constrained model predictive control, AIChE Journal, 45, 1743-1752, 1999.

[21] Vargas, A.N.; Costa, E.F.; do Val, J.B.R. (2013). On the control of Markov jump linear systems with no mode observation: application to a DC motor device, Int. J. Robust Nonlinear Control, 23, 1136-1950, 2013.

[22] Vargas, A. N.; do Val, J.B.R. (2010). Average cost and stability of time-varying linear systems, IEEE Trans. Autom. Control, 55, 714-720, 2010.

[23] Wang, S.; Yu, M.; Sun, X. (2015). Robust $H_{\infty}$ control for time-delay networked control systems with probability constraints, IET Control Theory Appl., 9, 482-2489, 2015. 
[24] Yang, H.; Guo, M.C.; Xia, Y.; Cheng, L. (2018). Trajectory tracking for wheeled mobile robots via model predictive control with softening constraints, IET Control Theory Appl., 12, 206-214, 2018.

[25] Yan, J.; Bitmead, R.R. (2005). Incorporating state estimation into model predictive control and its application to network traffic control, Automatica, 41, 595-604, 2005.

[26] Zeilinger, M.N.; Raimondo, D.M.; Domahidi, A.; Morari, M.; Jones, C.N. (2014). Flocking of multi-agents with a virtual leader, Automatica, 50, 683-694, 2014. 